\title{
MICROVARIABILITY OF S5 0716+714, A $\gamma$-RAY BLAZAR
}

\author{
R. NESCI , E, MASSARO, M. MAESANO AND F. MONTAGNI \\ Istituto Astronomico, Universita" "La Sapienza", Roma, ITALY \\ F. D'ALESSIO \\ Osservatorio Astronomico Collurania, Teramo, ITALY \\ AND \\ G. TOSTI AND M. LUCIANI \\ Osservatorio Astronomico, Universita' di Perugia, Perugia \\ ITALY
}

\section{Introduction}

Like many radio-selected blazars, $0716+714$ shows a high level of variability on different time scales, as short as a few days (e.g. Wagner et al 1996, Ghisellini et al. 1997). The mechanism of the emission in the optical band, in the general scheme of a relativistic plasma jet highy collimated toward the observer, is generally believed to be synchrotron radiation from electrons in a strong magnetic field. Our monitoring of S5 $0716+714$ is aimed to clarify whether the flux variations are chromatic or achromatic. In the first case, one could guess that variations in the spectrum of the injected electrons are responsible for the flux variations; in the second case, geometrical effects like small changes in the angle between the jet and the line of sight could be more likely (Wagner et al. 1993).

\section{Observations and data analysis}

Photometry was carried out with several telescopes: the $0.4 \mathrm{~m}$ automatic telescope of the Perugia University, the $0.5 \mathrm{~m}$ telescope at Vallinfreda (Roma), the $0.7 \mathrm{~m}$ telescope of the Astronomical Institute and IAS-CNR at Monte Porzio, the $0.7 \mathrm{~m}$ telescope of the Collurania-Teramo Observatory. In our search for intranight variations we generally used different telescopes, each one observing in a single filter, in order to sample each band in the most tight manner. Besides the standard Johnson-Cousins B,V,R,I filters, also 
TABLE 1. Observations log

\begin{tabular}{llllllll}
\hline JD & Day & MP & VA & TE & PG & R mag. & $\Delta \operatorname{mag}(\max )$ \\
\hline $2,450,369.5$ & Oct 12 & - & R & 99 & - & 13.6 & 0.00 \\
$2,450,396.4$ & Nov 08 & 80 & B & - & - & 13.3 & 0.10 \\
$2,450,400.4$ & Nov 12 & 80 & B & - & V & 13.5 & 0.17 \\
$2,450,401.4$ & Nov 13 & - & - & 86 & V,R,I & 13.7 & 0.16 \\
$2,450,424.4$ & Dic 06 & 86 & - & B & - & 14.1 & 0.25 \\
$2,450,425.4$ & Dic 07 & 86 & - & B & - & 14.3 & 0.00 \\
$2,450,465.4$ & Gen 16 & - & I & B & - & 13.6 & 0.09 \\
$2,450,511.4$ & Mar 03 & - & B,I & - & I & 13.9 & 0.00 \\
$2,450,512.4$ & Mar 04 & - & B,I & - & I & 13.9 & 0.17 \\
$2,450,520.4$ & Mar 12 & - & B,I & - & - & 13.9 & 0.13 \\
\hline
\end{tabular}

the 80, 86 and 99 narrow-band filters of the Arizona system (Johnson and Mitchell 1975) were used.

The main observational results of the intranight monitoring may be summarized as follows:

a) the luminosity of $0716+714$ was generally variable with an amplitude of at least several hundredths of magnitude on time scales of a few hours.

b) whenever the flux variation was substantially greater than our measuring accuracy (typically $0.02 \mathrm{mag}$ ), the slope of the light-curve was steeper at shorter wavelengths.

c) when we could observe both the raising and the falling branches of a local maximum we found that there was no plateau and the raising and falling times were substantially equal.

The dependence of the spectral index on the flux variations suggests that the most likely mechanism at work is a localized injections of energy in the form of fresh and rapidly cooling relativistic particles, superimposed to a steadier emission, possibly coming from larger regions. The lack of a plateau in the local maxima may be used to put constraints on the structure of the emitting region in the framework of SSC models, which will be discussed elsewhere.

\section{References}

Ghisellini G., Villata M., Raiteri C.M., et al. 1997, A\&A in press. Johnson H.L., Mitchell R.I., 1975, Re. Mexicana Astron. Astrophys. 1, 299 Wagner, S.J., Witzel, A., Krichbaum, T.P., et al. 1993, A\&A 271,344.

Wagner, S.J., Witzel, A., Heidt, J., et al., 1996, AJ, 111, 2187 